\title{
Rút bài vì sử dụng dữ liệu trái phép
}

sciencevietnam

SSHPA (30-09-2021) - Tạp chí International Journal of Older People Nursing đã ra quyết định rút bài báo "Co-designing technology with people with dementia and their carers: Exploring user perspectives when co-creating a mobile health application" vì lý do khá đặc biệt: Tác giả duy nhất của bài sử dụng dữ liệu không được phép, và không ghi nhận vai trò của các nhà nghiên cứu và nguồn tài trợ khác $[1,2]$.

Wiley Online Library Search

International Journal of

Older People Nursing

THIS ARTICLE HAS BEEN RETRACTED

Retracted: Co-designing technology with people with dementia and their carers: Exploring user perspectives when co-creating a mobile health application

(9 Retraction(s) for this article $\wedge$

Retracted: Co-designing technology with people with dementia and their carers: Exploring user perspectives when co-creating a mobile health application by O'Connor S

Volume 16, Issue 4, International Journal of Older People Nursing | First Published online: June 18, 2021

Siobhan O'Connor BSC CIMA CBA, BSC RN FHEA

First published: 14 December 2019 | https://doi.org/10.1111/opn.12288 | Citations: 6

Funding information:

The author gratefully acknowledges funding from the Burdett Trust for Nursing who supported this research study.

RetractionWatch cho biết, tạp chí International Journal of Older People Nursing đã quyết đinh rút bài sau khi nhận được thông tin từ trường đại học University of Edinburgh. Tuy nhiên, tạp chí đã không thể liên lạc trao đổi với tác giả duy nhất của bài [3].

Theo thông báo rút bài [2], trong thời gian làm giảng viên tại University of Edinburgh, tác giả chính bài báo đã sử dụng dữ liệu thuộc sở hữu của University of Glasgow mà cô có được khi còn là nghiên cứu sinh tại đây.

Vụ việc cho thấy nhiều vấn đề trong việc bình duyệt bài báo như quá trình kiểm tra dữ liệu và quyền sử dụng dữ liệu cần được chú ý hơn. Bên cạnh đó, mặc dù quá 
trình rút bài diễn ra nhanh chóng giữa tạp chí và University of Edinburgh, việc không liên lạc được với tác giả chính cũng cho thấy quá trình rút bài vẫn cần thay đổi để có thể đảm bảo công bằng cho các bên liên quan [4].

\section{Tài liệu tham khảo:}

[1] O'Connor, S. (2021). Retracted: Co-designing technology with people with dementia and their carers: Exploring user perspectives when co-creating a mobile health application. International Journal of Older People Nursing, 15(3), e12288.

[2] IJOPN. (2021). Retracted: Co-designing technology with people with dementia and their carers: Exploring user perspectives when co-creating a mobile health application by O'Connor S. International Journal of Older People Nursing, 16(4), e12392.

[3] Marcus, A. (2021). Award-winning nursing researcher's paper retracted for 'failure to acknowledge the contribution of other researchers and the funding source'. RetractionWatch. URL: https://retractionwatch.com/2021/o7 /20/award-winning-nursing-researchers-paper-retracted-for-failure-toacknowledge-the-contribution-of-other-researchers-and-the-funding-source/

[4] Vuong, Q. H. (2020). Reform retractions to make them more transparent. Nature, 582(7811), 149

Last modified: 9/30/2021 4:19 PM Views: 20

\section{Bài liên quan:}

- Không ăn được thì đạp đổ

- Scientometrics rút bài báo sử dung dư liệu từ danh sách Beall

- Bài nghiên cứu phải xin rút bài tới hai lần

- Tác giả Việt nêu quan điểm về việc rút bài báo khoa học trên Nature

- Lỗi tại Excel 